\title{
Direct Brow Lift Combined with Suspension of the Orbicularis Oculi Muscle
}

\author{
Jeong Woo Lee ${ }^{1}$, Byung Chae Cho ${ }^{1}$, Kyung Young Lee ${ }^{2}$ \\ ${ }^{1}$ Department of Plastic and Reconstructive Surgery, Kyungpook National University School of Medicine, Daegu; ${ }^{2}$ Dr. Lee's Aesthetic Plastic \\ Surgical Clinic, Daegu, Korea
}

Background Although the conventional direct brow lift operation provides a simple means of managing lateral brow ptosis, the scars produced have been unacceptable. However, using the modifications proposed here, scarring showed remarkable improvement. This article reviews our experiences with the presented technique, mainly with respect to postoperative scarring. Methods Measured amounts of supra-eyebrow skin and subcutaneous fat were excised en bloc in the conventional manner under 'hyper-hydrated' local infiltration anesthesia. The lower flap and the edge of the upper flap were undermined above the muscular plane, and the orbicularis oculi muscle was directly suture-plicated and suspended upward to the distal frontalis muscle. Skin closure was performed in a basic plastic surgical manner.

Results From April 2007 to April 2012, a consecutive series of 60 patients underwent surgery using the above method. The average width of the excised skin was $8 \mathrm{~mm}$ (range, 5-15 mm) at the apex of the eyebrow. Preoperative complaints were resolved without occurrence of significant complications. The surgical scars showed remarkable improvement and were negligible in the majority of the cases.

Conclusions The direct brow lift operation combined with plication/suspension of the superior and lateral portion of the orbicularis oculi muscle provides a simple, safe, and predictable means of correcting lateral brow ptosis. The scars were acceptable to all of the patients. For proper management of the frontalis tone, upper blepharoplasty and/or repair of eyelid levator function must be considered in addition to brow lift procedures.

Keywords Rhytidoplasty / Forehead / Cicatrix
Correspondence: Kyung Young Lee Dr. Lee's Aesthetic Plastic Surgical Clinic, 3F, 2403 Dalgubeol-daero, Suseong-gu, Daegu 706-817, Korea Tel: +82-53-756-3433

Fax: +82-53-756-5231

E-mail: walker3433@naver.com

Received: 16 Mar 2013• Revised: 26 May 2013 • Accepted: 28 May 2013

pISSN: 2234-6163 • elSSN: 2234-6171 • http://dx.doi.org/10.5999/aps.2013.40.5.603 • Arch Plast Surg 2013;40:603-609

No potential conflict of interest relevant to this article was reported.

\section{INTRODUCTION}

Lateral eyebrow ptosis, the earliest manifestation of the aging forehead [1], gives the face a tired look and accentuates deformities of the upper eyelids. A close relationship exists among the frontalis muscle tone, eyebrow position, and upper eyelid hanging. In some cases, decreased function of the levator muscle of the upper eyelid or the patient's habits can also be related to frontalis tone. In cases of ptotic eyebrow, the underlying orbicularis muscle is also descended with the skin and subcutaneous fat. The accumulated results are the typical characteristics of the aged upper face, including forehead wrinkles, low-lying eyebrows, hanging of the upper eyelids causing an impaired visual field, accentuated crow's feet, and, sometimes, eczema in the lateral canthus.

In women, the eyebrow is positioned above the orbital rim and

Copyright $($ C 2013 The Korean Society of Plastic and Reconstructive Surgeons

This is an Open Access article distributed under the terms of the Creative Commons Attribution Non-Commercial License (http://creativecommons.org/

licenses/by-nc/3.0/) which permits unrestricted non-commercial use, distribution, and reproduction in any medium, provided the original work is properly cited.

www.e-aps.org 
is arched, with its apex between the lateral limbus and lateral canthus; in men, it is flatter and lower [2]. Freund and Nolan [3] have suggested that the medial eyebrow should be located at or below the supraorbital rim, not above it, and that the eyebrow should be shaped with its highest point located lateral to the pupil ("apex lateral slant").

In Korea, approximately $50 \%$ to $60 \%$ of patients have no superior palpebral folds $[4,5]$, but performance of the brow lifting procedure and creation of superior palpebral folds during the same operation session is not easy. In many cases, creation of a 'double eyelid' by upper blepharoplasty can bring the lateral eyebrow down to a more ptotic level due to the loss of frontalis muscle contraction to clear the impaired visual field, which is easily detected during preoperative simulation of the palpebral folds using a stick (unfolded paperclip). In order to obtain optimum cosmetic results, we corrected brow ptosis first, and then performed upper blepharoplasty with or without repair of the eyelid levator function after adequate subsidence of operative edema. In addition, many women obtained an eyebrow tattoo and underwent shaving of the ptotic lateral eyebrow in order to camouflage its ptotic shape and/or conceal sparse hairs. This article reviews our experiences with the presented technique, mainly with respect to postoperative scarring.

\section{METHODS}

\section{Patients}

Patients whose lateral eyebrows were located on or below the supraorbital rim with a relaxed frontalis muscle and closed-eye state were good candidates, and young patients with congenitally low-set eyebrows were also possible candidates. On the other hand, patients with combined diminished levator function required more comprehensive preoperative evaluation.

However, when a patient had a ptotic medial end of the eyebrow that needed lifting, or an unacceptable aggravation of wrinkles at the root of the nose with the eyes closed and a relaxed frontalis tone, the case was excluded. Other physical and psychological contraindications were also assessed.

From April 2007 to April 2012, a total of 60 patients (female 51 , male 9) underwent this procedure, and their ages ranged from 15 to 80 years old (average 54, most between 50 and 70 ). Six patients were younger than 25 years old. One of them had a drooping left eyebrow after multiple cranial bone shavings via a bicoronal approach due to fibrous dysplasia associated with McCune-Albright syndrome, and the others had congenitally lowset eyebrows.

\section{Preoperative measurements}

In the preoperative frontal view photogram, the interpupillary line was arrayed horizontally; the symmetry of the eyebrows was checked, and any significant asymmetry was recorded.

In the sitting position with the eyes closed, and with the frontalis muscle fully relaxed, a vertical line was drawn through the apex of the eyebrow arch (Fig. 1A). A starting point was marked at the apex of the eyebrow on the vertical line, and the apex was lifted using the thumb while the pen was fixed in position to the height that the eyebrow shape is acceptable; the second point was then marked (Fig. 1B, C). During this process, in order to avoid measurement errors, the pen and the pen-holding hand must not move. After this stage, the patient could imagine the postoperative eyebrow and eyelid shape using a hand mirror. The distance between the two marks in the vertical line would be the width of the skin flap to be excised at the apex (Fig. 1C).

\section{Surgical design}

In the supine position, after adequate skin preparation and draping, the lower line of the design was drawn along the supraciliary line. This line could be within the eyebrow itself or the tattoo, if needed, according to preoperative discussion with the patient. The eyebrow below this line would be the future eyebrow. The

\section{Fig. 1. Measurement of lateral brow ptosis}

\section{(A) Fully relaxed frontalis tone. (B) The first point was marked at the vertical line and the apex was lifted using the thumb (white arrow), while the pen was fixed in position until an adequate level compatible with the shape of the eyebrow was reached; the second point was then marked. (C) The distance between the two marks (white arrows) was measured using a ruler.}
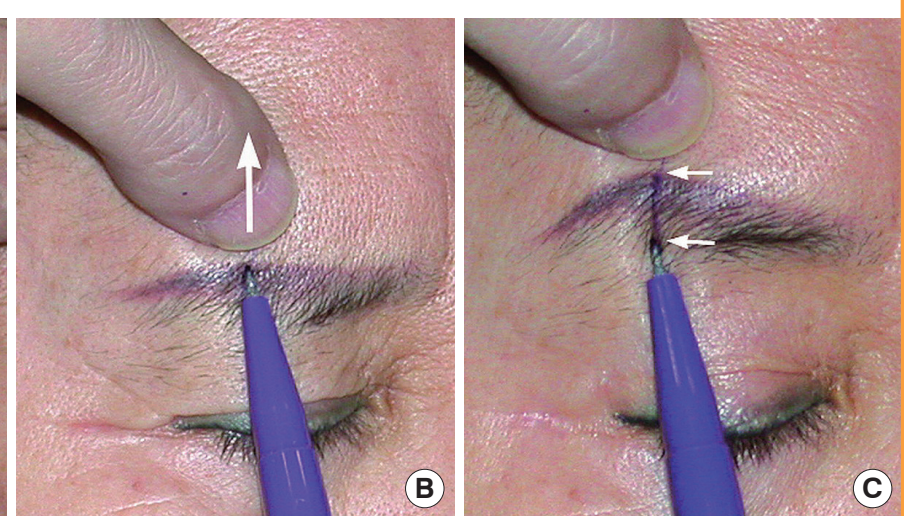
upper line of the design was drawn to represent the measured value at the apex and the results of preoperative photo analysis. If more lift in the lateral 'crow's feet' area was needed, the lateral part of the design was modified to the scalpel blade-like extension [6]; finally, the location of the supraorbital notch (or foramen) was identified by palpation, and the expected courses of the supraorbital and supratrochlear nerves were marked (Fig. 2A).

\section{Surgical technique}

Local infiltration of $1 \%$ lidocaine with 1:200,000 epinephrine was performed using a 30-gauge, one-inch-long needle. The infiltration was mainly performed into the subcutaneous fat layer until definite swelling was obtained (approximately $5 \mathrm{~mL}$ per side). The skin incision was performed using a No. 15 blade with deepening into the full thickness of the 'hydro-ballooned' fat layer. Care must be taken in the sensory nerve-bearing area in order not to inadvertently deepen through the muscle layer. After completing the incision, en bloc excision of the skin-fat flap was started from the lateral end using blunt, small, curved Metzenbaum scissors for dissection just above the muscle layer. This dissection was relatively simple and bloodless due to the 'hyper-hydration' and the effect of epinephrine. In most cases, near the medial end, the flap was easily elevated by gentle traction, possibly because 'hyper-hydration' caused loosening of the fatty tissue. This elevation provided additional protection to the sensory nerves.

At the apical area, the edge of the lower flap was hooked and dissected caudally using small curved scissors between the fat and muscle layers with a spreading motion to a distance of approximately $10 \mathrm{~mm}$ or more, as required. The dissection was extended laterally and medially along the full length of the incision. Medially, the width of the dissection was gradually narrowed. In order to avoid damage to the hair follicles or nerves, dissection did not involve violation of the fatty layer or muscle layer. Fatty muscle infiltration was sometimes encountered, especially in older patients. The upper flap margin was dissected to a width of approximately $5 \mathrm{~mm}$ just above the muscle plane in order to facilitate muscular suspension to the frontalis muscle and skin closure (Fig. 2B).

After completing the dissection, the drooping orbicularis oculi muscle was grasped using a toothed forceps and pulled cephalically at the apical area. The width and level of plication/ suspension was then determined; in general, the width was approximately 10 to $15 \mathrm{~mm}$. First, a vertical suture-plication/ suspension was applied to the distal frontalis muscle at its apex using a 5-0 Vicryl suture (Ethicon Inc., Somerville, NJ, USA), and two narrower medial sutures were added to the distal frontalis muscle. The lateral portion of the orbicularis oculi muscle

\section{Fig. 2. Surgical design and procedure}

(A) Surgical design. The dotted vertical lines reflect the expected courses of sensory nerves. (B) After skin flap excision and completion of undermining of the lower flap and edge of the upper flap. The orbicularis oculi muscle (white arrows indicate the upper border of the orbicularis muscle) and distal portion of the frontalis muscle (yellow arrows indicate distal frontalis, blue arrow indicates superficial temporal fascia [STF]) were exposed. (C) After suture-plication/suspension of the orbicularis oculi muscle to the distal portion of the frontalis muscle in the apical and medial areas with three 5-0 Vicryl stitches, lateral suspension to the STF, which is continuous with the frontalis muscle (white arrows). (D) The skin was closed in two layers with 5-0 Vicryl and 6-0 nylon.
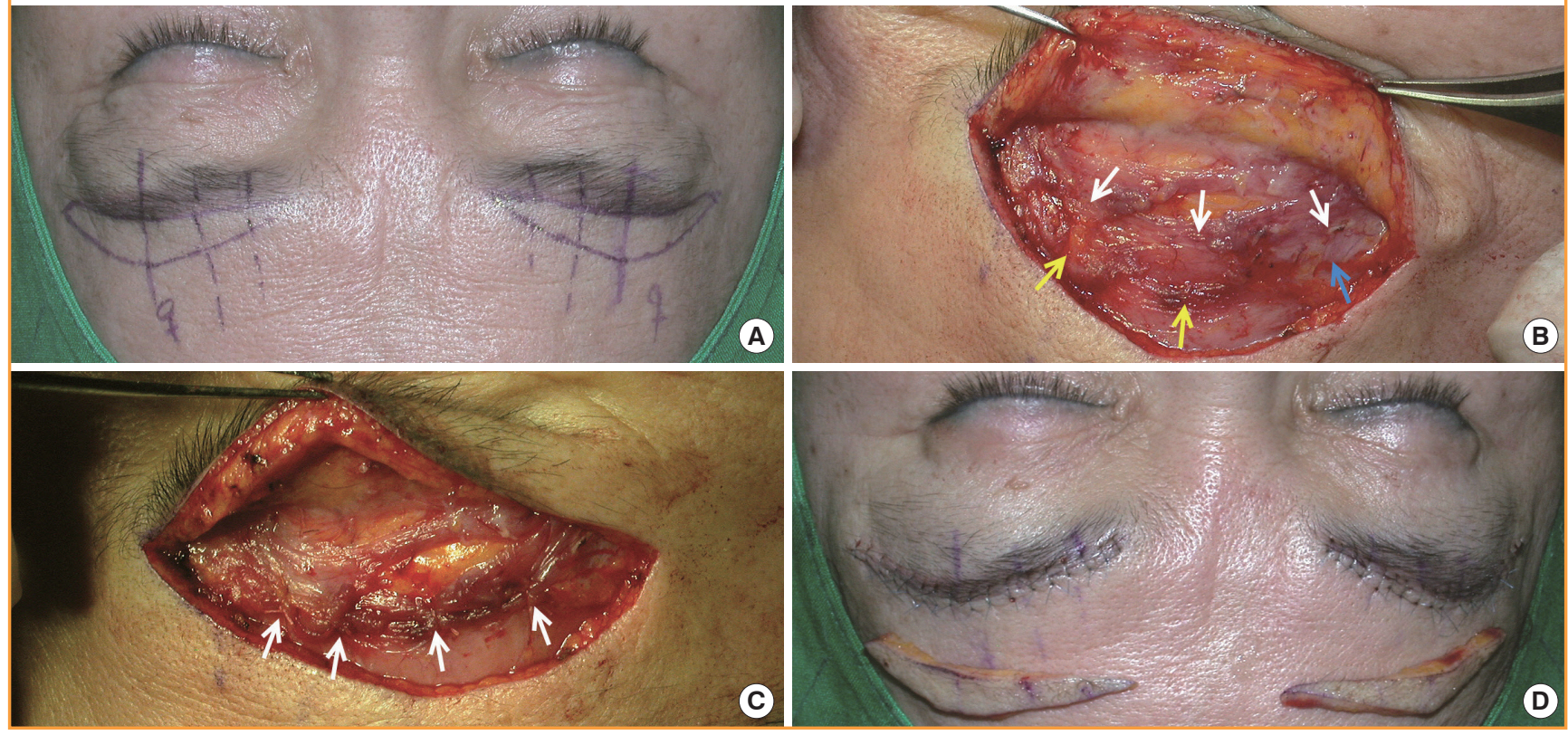
was then plicated and suspended from the fascial structure laterally, extending from the frontalis muscle, that is, the superficial temporal fascia (STF) (Fig. 2C). Using plication sutures, the excised gap was then narrowed, and, after confirming hemostasis, the skin was closed in two layers with 5-0 Vicryl and 6-0 nylon simple interrupted sutures according to basic plastic surgical principles (Fig. 2D). Mild pressure dressing with gauze and tape was applied. The wound was checked on the first postoperative day, and the stitches were removed on the fifth postoperative day.

\section{RESULTS}

The average width of the excised skin flaps was $8 \mathrm{~mm}$ (range, $5-15 \mathrm{~mm}$ ). The most frequent preoperative complaints were a tired-looking appearance and lateral heaviness. Others included accentuated 'crow's feet', forehead wrinkles, eczematous erosion at the lateral canthus, and, sometimes, eyebrow shape. The average follow-up period was 9 months (range, 1-36 months). The characteristic reddened phase of the early surgical scars (between 1 and 2 months postoperatively) was not recognized, and most of the patients felt no special need to camouflage the scar in their ordinary life even before 1 month postoperatively.

The issues about which patients had complained showed remarkable improvement in the majority of the patients. In some patients with tattooed eyebrows, portions of the tattoos were resected and required revision later. There was no occurrence of hair loss in the dissected eyebrow area or sensory changes of the forehead. Revisionary brow lift operation after successive upper blepharoplasty was performed in two patients during the early period. There was no case of infection of the surgical wound or significant hematoma. One patient underwent partial wound dehiscence at 3 days after stitch removal due to an unknown cause. The surgical scars were accepted by all of the patients, and were nearly invisible in most, other than some patients with thick sebaceous pigmented skin or young patients.

\section{Case 1}

A 54-year-old female presented with ptotic lateral eyebrows and a tired-looking appearance. She had artificial tattooed eyebrows. The supra-eyebrow crescentic flap along the whole length was excised, and its width at the apical area was $8 \mathrm{~mm}$ on both sides. The surgical scars were unrecognizable in the 2-year postoperative photogram. The thickness of the upper lids was thinned (Fig. 3).

Fig. 3. A 54-year-old female with ptotic lateral eyebrows

(A) Preoperative view. (B) Two years after the brow lift operation.
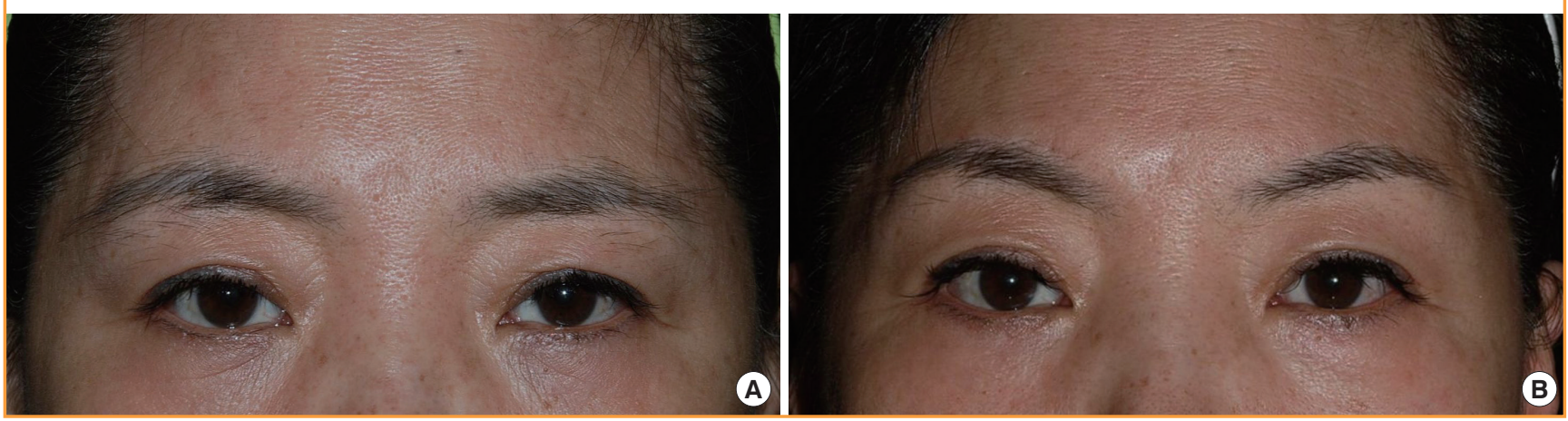

\section{Fig. 4. A 63-year-old female with weak levator function}

(A) The ptotic lateral eyebrows were camouflaged by tattoos, and her inborn suprapalpebral folds were covered by hanging lid skin. Frontal furrows compensated for the ptotic eyebrows, and the levator function was weak. (B) Four months after a direct brow lift. (C) Close-up view of the surgical scar.
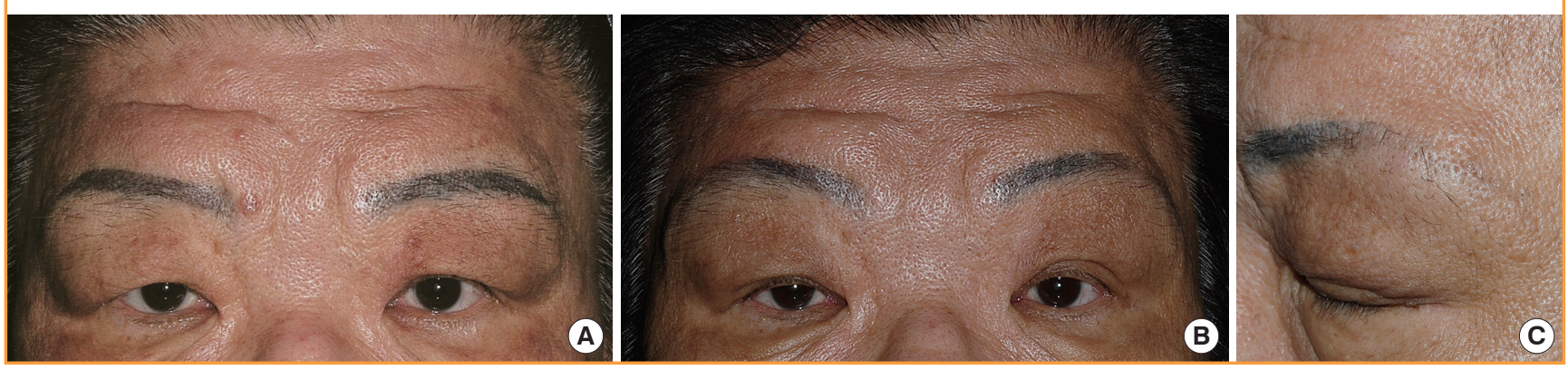
Fig. 5. A 41-year-old male with prominent frontalis tone

(A) Preoperative views. (B) Six months after the brow lift operation (a width of $13 \mathrm{~mm}$ was excised at the apex) and successive upper blepharoplasty. The frontalis showed a remarkable decrease in tone.
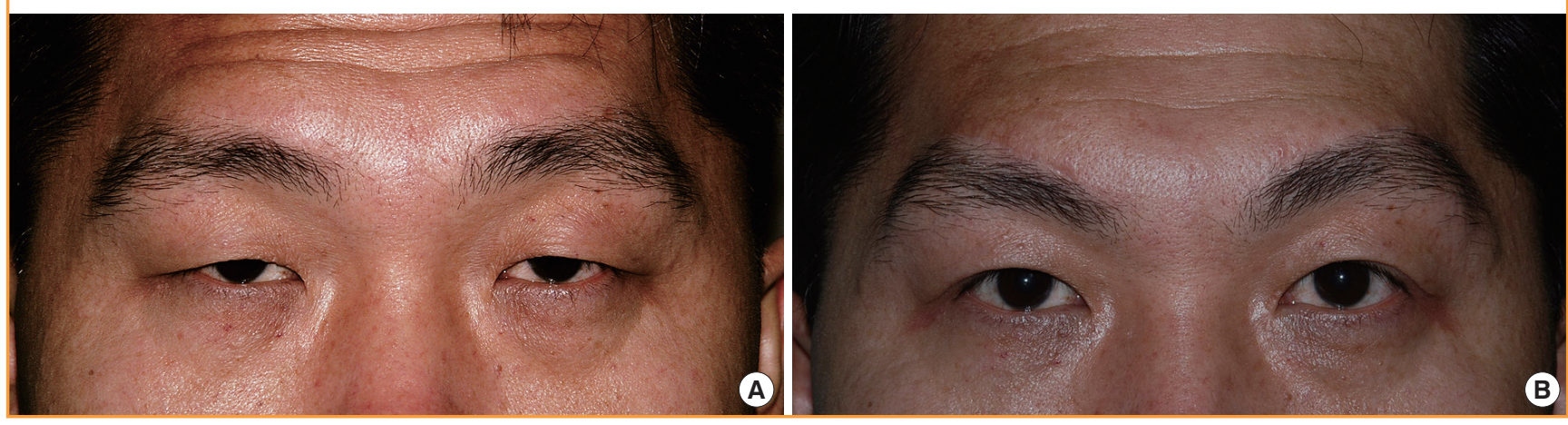

\section{Case 2}

A 63-year-old female presented with frontal heaviness and frequent erosion at the lateral canthal areas. The ptotic lateral eyebrows were camouflaged by tattoos, and her inborn suprapalpebral folds were covered by hanging lid skin. She also had weak levator function. Frontal furrows compensated for the ptotic eyebrows and weak levator function. A 10-mm skin strip, including part of a tattoo, was excised from each side. In Fig. 4B, her eyebrows are too high due to the compensatory contraction of the frontalis muscle for her weak levator muscle function; however, they fell after upper blepharoplasty with enforced levator function. The scar was acceptable even in the presence of postoperative persistent increased frontalis tone in a close-up view (Fig. 4).

\section{Case 3}

A 41-year-old male presented with prominent frontalis tone. He also experienced frequent frontal headaches. He had ptotic eyebrows, possibly combined with congenitally low-set eyebrows and hanging of the upper eyelids. The eyelid levator function was good. He underwent the brow lift operation (a width of 13 $\mathrm{mm}$ was excised at the apex), followed by upper blepharoplasty two weeks later. The frontalis showed a remarkable decrease in tone. His preoperative frontal headache symptoms also disappeared. He still had some hanging of the upper eyelids but declined further surgery. In Fig. 5B, the focal depressed scar at the medial area of the right eyebrow was caused by partial wound dehiscence at 3 days after stitch removal due to an unknown cause (Fig. 5).

\section{DISCUSSION}

Surgical procedures used to improve the effects of aging on the forehead vary in terms of the positions of incisions and planes of dissection on the scalp and forehead. Nowadays, the conventional bicoronal technique has been largely replaced by the endoscopic technique and other less invasive procedures. After 1992, the endoscopic lift was widely accepted due to successful outcomes [7]. However, many authors have felt the need to fix the elevated brow in position in order to prolong the effect. Pascali et al. [8] proposed the application of an Endotine Ribbon (Coapt Systems Inc., Palo Alto, CA, USA), and Marchac et al. [9] suggested using fibrin glue to fix the brow in the proper position during an endoscopic lift. Other authors have also reported the need to fix lifted brows in position in order to prolong results $[10,11]$. Chiu and Baker [7] reported a striking decline in the overall number of endoscopic brow lift procedures performed by New York plastic surgeons between 1997 and 2001, and that only $50 \%$ of plastic surgeons were pleased with longterm results. Freund and Nolan [3] reported that standard open and endoscopic brow lift operations frequently result in unsatisfactory eyebrow height and shape. A transpalpebral approach $[12,13]$ and suture suspension methods $[14,15]$ have also been reported; however, the long-term effects of these approaches are uncertain.

Some authors have reported on the use of botulinum toxin type A to weaken the depressing power of the orbicularis oculi muscle with brow elevation due to the counter-effect of the frontalis muscle $[16,17]$. However, the effect of botulinum toxin is of short duration, and the amount of elevation is limited. Other authors have reported on internal suture plication or suspension of the muscle as an adjunct to blepharoplasty $[18,19]$.

Supraciliary excision of the skin for direct brow elevation has been described by Passot [20], Castanares [21], and Har-Shai et al. [6]. Despite its ease and efficacy for correction of lateral brow ptosis, this technique leaves unacceptable surgical scars.

The modifications described here were devised with a focus on reducing postoperative scarring. A measured amount of 
supraciliary skin and subcutaneous fat was excised. Flaps were dissected just above the muscle layer, and the superior portion of the orbicularis muscle was directly suture-plicated and suspended from the distal portion of the frontalis muscle. Use of these procedures resulted in reduced tension at the skin suture line due to the undermining of flap edges, and plication/suspension also reduced tension to some extent. Even in the patient with definite postoperative remnant frontalis contraction due to pre-existing weakness of the eyelid levator function or another cause, the appearance of the surgical scar was unaffected (Fig. 4). This should be because the frontalis contraction gathers the overlying suture line, rather than spreading it, due to its re-enforced connection with the underlying orbicularis oculi muscle. In the lateral one-third area, where direct suspension from the frontalis muscle is unavailable, the appearance of the scar does not differ from that of the medial two thirds. Lateral plication contributes to spreading of thick lateral eyelid tissue and has a suspending effect on the STF (Fig. 2B, C). While plicating this area, care should be taken not to damage the frontal branch of the facial nerve; however, the upper limit of plication is usually sufficiently below the expected course of this branch, and on no occasion was it injured during surgery on the 60 consecutive cases.

We believe that the plicated portion of the orbicularis oculi muscle will lose volume and that this will result in a decrease in the constricting power of the orbicularis muscle. However, according to the Frank-Starling mechanism, the spreading and widening of the lower portion of the orbicularis oculi muscle by plication and suspension will increase its contractile power. Accordingly, we are unable to explain net changes in the constricting power of the orbicularis oculi muscle.

During the preoperative measurement procedure, in order to determine the 'real' amount of ptosis, the patient's eyes should be closed and the frontalis muscle fully relaxed. In view of the additional decrease in frontalis tone after upper blepharoplasty, this is of considerable importance. Even in patients with a weaker eyelid levator or dermatochalasis, the skin flap is excised to the same amount measured using the method mentioned above (Figs. 4, 5). The level of the eyebrows, which is higher due to the remnant frontalis tone, will fall down after successive blepharoplasty with or without strengthening of the eyelid levator function. Two patients treated during the early period required a secondary direct brow lift procedure after successive upper blepharoplasty, and the causes were an additional decrease in frontalis tone after upper blepharoplasty and/or an underestimation of the amount of brow ptosis during the initial procedures. These cases served as a reminder of the importance of frontalis tone relaxation when taking preoperative measurements.

Nearly half of the 60 consecutive patients, especially those aged over 60 , underwent the brow lift procedure only and declined to undergo successive upper blepharoplasty to further improve cosmesis or function. Nonetheless, satisfaction levels were high due to a feeling of lightness when opening the eyes and of an improved shape of the periorbital areas after the operation.

Booth et al. [22] reported an incidence of altered sensation over the forehead of $74 \%$ (32/43 brows). However, we encountered no such case. We believe that it is a benefit of the "hyperhydrated' local infiltration anesthesia. The 'hyper-hydrated' thickened fatty layer facilitated easier dissection without injuring the nerves.

It should be noted, that the method described here is limited in terms of elevating the medial ends of the eyebrows, and could possibly worsen wrinkles at the nasal root by decreasing frontalis tone.

\section{REFERENCES}

1. Knize DM. An anatomically based study of the mechanism of eyebrow ptosis. Plast Reconstr Surg 1996;97:1321-33.

2. Larrabee WFJ, Makielski KH, Henderson JL. Surgical anatomy of the face. 2nd ed. Philadelphia: Lippincott Williams \& Wilkins; 2004.

3. Freund RM, Nolan WB 3rd. Correlation between brow lift outcomes and aesthetic ideals for eyebrow height and shape in females. Plast Reconstr Surg 1996;97:1343-8.

4. Park YK, La TK. Interpupillary distance and inter-inner canthal distance of Konans. J Korean Ophthalmol Soc 1975;16: 371-4.

5. Park DM, Song JW, Han KH, et al. Anthropometry of normal Korean eyelids. J Korean Soc Plast Reconstr Surg 1990; 17:822-41.

6. Har-Shai Y, Gil T, Metanes I, et al. Brow lift for the correction of visual field impairment. Aesthet Surg J 2008;28:512-7.

7. Chiu ES, Baker DC. Endoscopic brow lift: a retrospective review of 628 consecutive cases over 5 years. Plast Reconstr Surg 2003;112:628-33.

8. Pascali M, Gualdi A, Bottini DJ, et al. An original application of the Endotine Ribbon device for brow lift. Plast Reconstr Surg 2009; 124:1652-61.

9. Marchac D, Ascherman J, Arnaud E. Fibrin glue fixation in forehead endoscopy: evaluation of our experience with 206 cases. Plast Reconstr Surg 1997;100:704-12.

10. Gold AH, Bernard RW, Hamas RS, et al. Brow lift fixation. Aesthet Surg J 2003;23:217-9.

11. Ellenbogen R, Youn A, Svehlak S, et al. Transcoronal brow lift fixation: a 2-point dermis-to-skull fibrous scar adhesion. 
Aesthet Surg J 2004;24:261-4.

12. Codner MA, Kikkawa DO, Korn BS, et al. Blepharoplasty and brow lift. Plast Reconstr Surg 2010;126:1e-17e.

13. Cohen BD, Reiffel AJ, Spinelli HM. Browpexy through the upper lid (BUL): a new technique of lifting the brow with a standard blepharoplasty incision. Aesthet Surg J 2011;31: 163-9.

14. Erol OO, Sozer SO, Velidedeoglu HV. Brow suspension, a minimally invasive technique in facial rejuvenation. Plast Reconstr Surg 2002;109:2521-32.

15. Lewis JR Jr. A method of direct eyebrow lift. Ann Plast Surg 1983;10:115-9.

16. Fagien S. Temporary management of upper lid ptosis, lid malposition, and eyelid fissure asymmetry with botulinum toxin type A. Plast Reconstr Surg 2004;114:1892-902.
17. Ahn MS, Catten M, Maas CS. Temporal brow lift using botulinum toxin A. Plast Reconstr Surg 2000;105:1129-35.

18. Zarem HA, Resnick JI, Carr RM, et al. Browpexy: lateral orbicularis muscle fixation as an adjunct to upper blepharoplasty. Plast Reconstr Surg 1997;100:1258-61.

19. Niechajev I. Transpalpebral browpexy. Plast Reconstr Surg 2004;113:2172-80.

20. Passot R. Chirurgie esthetique pure: technique et resultats. Paris: Gaston Doin et Cie; 1931.

21. Castanares S. Forehead wrinkles, glabellar frown, and ptosis of the eyebrows. Plast Reconstr Surg 1964;34:406-13.

22. Booth AJ, Murray A, Tyers AG. The direct brow lift: efficacy, complications, and patient satisfaction. $\mathrm{Br} \mathrm{J}$ Ophthalmol 2004;88:688-91. 